\title{
Patient-reported treatment delays in breast cancer care during the COVID-19 pandemic
}

\author{
Elizabeth Lerner Papautsky ${ }^{1}$ (1) $\cdot$ Tamara Hamlish $^{2}$
}

Received: 6 June 2020 / Accepted: 20 July 2020 / Published online: 9 August 2020

○) Springer Science+Business Media, LLC, part of Springer Nature 2020

\begin{abstract}
Purpose The coronavirus disease (COVID-19) pandemic has had a profound impact on cancer care in the US Guidelines focused on the management of COVID-19, rather than healthcare needs of breast cancer patients requiring access to crucial services. This US survey of breast cancer survivors characterizes treatment delays early period in the pandemic.

Methods We developed a survey and administered it to 609 adult breast cancer survivors in the US. We used snowball sampling with invitations distributed via social media. We used logistic regression to select a model of delay from a pool of independent variables including race, cancer stage, site of care, health insurance, and age. We used descriptive statistics to characterize delay types.

Results Forty-four percent of participants reported cancer care treatment delays during the pandemic. Delays in all aspects of cancer care and treatment were reported. The only variable which had a significant effect was age $(97(.95,99), p<0.001)$ with younger respondents $(M=45.94, S D=10.31)$ reporting a higher incidence of delays than older respondents $(M=48.98$, $S D=11.10$ ). There was no significant effect for race, insurance, site of care, or cancer stage.

Conclusions Our findings reveal a pervasive impact of COVID-19 on breast cancer care and a gap in disaster preparedness that leaves cancer survivors at risk for poor outcomes. Delays are critical to capture and characterize to help cancer providers and healthcare systems develop effective and patient-tailored processes and strategies to manage cases during the current pandemic wave, subsequent waves, and future disasters.
\end{abstract}

Keywords COVID-19 $\cdot$ Breast cancer $\cdot$ Treatment delays $\cdot$ Survey research

\section{Introduction}

The coronavirus disease (COVID-19) pandemic has had a profound impact on cancer care across the globe. Early data indicate increased risk of COVID-19 morbidity and mortality for cancer survivors $[1,2]$. Healthcare systems have demonstrated considerable variation in preparedness and capacity, as well as organizational structure and agility in responding to disruptions in cancer treatment $[1,3]$. Emerging guidelines for cancer care during the pandemic are working to address the increased demand on medical

Elizabeth Lerner Papautsky

elp@uic.edu

1 Department of Biomedical \& Health Information Sciences, University of Illinois at Chicago, 1919 W. Taylor St., Chicago, IL 60612, USA

2 University of Illinois Cancer Center, University of Illinois at Chicago, 818 S. Wolcott Ave., Chicago, IL 60612, USA resources and balance the risk of treatment delays against the potential exposure to the virus $[4,5]$. Many of these guidelines are based largely on clinical criteria and may not take into account the many factors that affect cancer outcomes, including the healthcare system factors outlined above, as well as factors such as social determinants of health and health inequities which have been exacerbated by the pandemic [6-8].

Delays in chronic disease care and treatment during a time of disaster have been well documented [9-12]. Disaster preparedness planning frequently focuses on the medical needs of those immediately affected by the disaster, with limited attention to the allocation of scarce healthcare resources for chronic disease management. However, interruptions in care due to disasters often result in increased rates of disease progression, pain, and excess mortality [13-15]. A recent study estimates 33,890 excess deaths among US cancer survivors over the age of 40 as a result of COVID-19 [16]. 
This investigation of the impact of the pandemic on breast cancer care in the US is intended to inform the allocation of healthcare resources and services during the recovery from the current COVID-19 pandemic, and support improved preparedness for disruptions due to future disasters. This national survey of breast cancer survivors examines multiple factors that contribute to disparities in access to cancer care to gain a comprehensive understanding of delays in breast cancer care during the early period of the pandemic.

\section{Methods}

We developed a 50-item survey to assess the impact of COVID-19 on breast cancer care. The survey was reviewed and piloted by two breast cancer survivors and one clinician. Data were collected using Research electronic data capture (REDCap) hosted at the University of Illinois at Chicago [17]. We employed the National Cancer Institute definition of cancer survivor, which considers a person to be a survivor from the time of diagnosis until the end of life [18]. We used snowball sampling to recruit adult ( $>18$ years old) breast cancer survivors in the US. Survey invitations were distributed via social media and email to breast cancer patient support networks.

This analysis focuses on the question of whether respondents experienced delays in cancer-related care or treatment and compares the demographic and clinical characteristics of those who reported delays and those who did not report delays.

All analyses used SPSS version 26 statistical software and Excel. Descriptive statistics of counts, proportions, means and standard deviations were calculated. Inferential statistics used were logistic regression. The study was reviewed by University of Illinois at Chicago's Institutional Review Board (Protocol \#2020-0371; Exemption Granted: 3/27/20).

\section{Results}

From 4/2/20 to 4/27/20, 609 respondents ( 1 male) completed the survey. Average age was $47.76(S D=10.86$, range 22-80, $M=46$ ) (see distribution in Fig. 1); average age at diagnosis was $43(S D=10.84) ; 46 \%$ of respondents were within 5 years of their most recent diagnosis. Respondents were racially diverse; $78 \%$ were white, $17 \%$ were black, and 3\% were Asian. The majority of respondents were non-Hispanic (95\%). Top three states of residence were Illinois (15\%), California (9\%), and Texas (8\%). More than half of respondents are currently receiving cancer treatment $(63 \%)$ and have employer-sponsored health insurance (67\%). Respondents receive cancer care at academic medical centers (45\%), physician's offices (26\%) or community hospitals (21\%).

Forty-four percent of participants responded Yes to the question: Has any aspect of your cancer care been delayed or interrupted due to the COVID-19 pandemic? We used a binary logistic regression model to select a best predictive model of delay (dichotomous) from a pool of independent variables including categorical variables (race, cancer stage, site of care, and health insurance) as well as age (continuous). There was no significant effect for race, insurance, site of care, or cancer stage. Reports of delays in care were slightly higher among White (46\%) and Asian (50\%) respondents, compared to Black respondents (36\%). Those with public insurance (Medicare, Medicaid) reported slightly lower rates of delay (36\%) compared with respondents with employer-sponsored health insurance (46\%) or who purchased private insurance (47\%). Delays based on cancer stage showed little variation: $44 \%, 46 \%$,
Fig. 1 Age distribution and descriptive statistics in sample of respondents

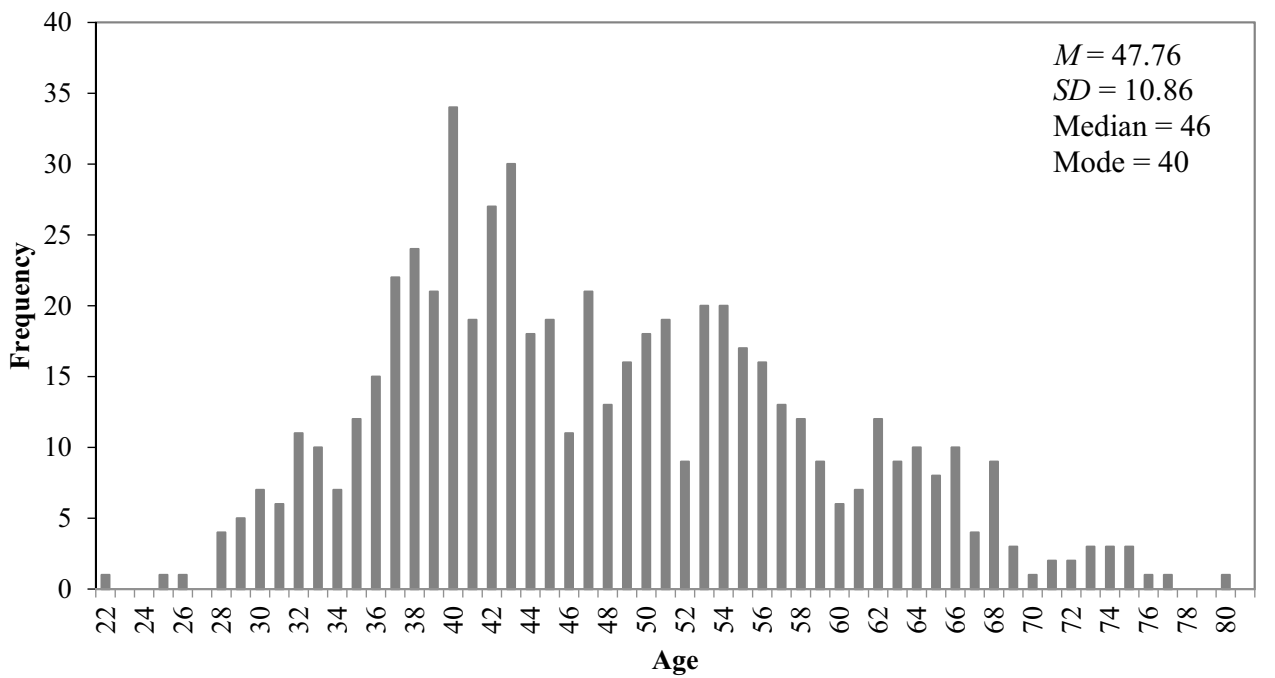

Age 
Table 1 Characteristics of respondents who experienced delays compared with those who did not $(n=554)$

\begin{tabular}{|c|c|c|c|c|c|c|c|c|}
\hline & & & \multicolumn{4}{|c|}{ Delay } & \multicolumn{2}{|c|}{$\begin{array}{l}95 \% \text { CI for } \\
\operatorname{Exp}(\beta)\end{array}$} \\
\hline & & & No & Yes & Sig & $\operatorname{Exp}(\beta)$ & Lower & Upper \\
\hline \multirow[t]{12}{*}{ Race } & American Indian or Alaska Native & Count & 2 & 1 & 0.81 & 0.66 & 0.02 & 19.40 \\
\hline & & $\%$ & $67 \%$ & $33 \%$ & & & & \\
\hline & Asian & Count & 7 & 7 & 0.82 & 0.78 & 0.09 & 6.71 \\
\hline & & $\%$ & $50 \%$ & $50 \%$ & & & & \\
\hline & Native Hawaiian or Pacific Islander & Count & 2 & 1 & 0.64 & 0.48 & 0.02 & 10.86 \\
\hline & & $\%$ & $67 \%$ & $33 \%$ & & & & \\
\hline & Black & Count & 66 & 37 & 0.51 & 0.52 & 0.08 & 3.53 \\
\hline & & $\%$ & $64 \%$ & $36 \%$ & & & & \\
\hline & White & Count & 238 & 204 & 0.80 & 0.79 & 0.12 & 5.11 \\
\hline & & $\%$ & $54 \%$ & $46 \%$ & & & & \\
\hline & Total & Count & 317 & 253 & & & & \\
\hline & & $\%$ & $56 \%$ & $44 \%$ & & & & \\
\hline \multirow[t]{12}{*}{ Site of care } & University/Academic Medical Center & Count & 142 & 127 & 0.17 & 1.69 & 0.80 & 3.57 \\
\hline & & $\%$ & $53 \%$ & $47 \%$ & & & & \\
\hline & Physician's Office & Count & 95 & 60 & 0.75 & 1.14 & 0.52 & 2.49 \\
\hline & & $\%$ & $61 \%$ & $39 \%$ & & & & \\
\hline & Veterans affairs hospital & Count & 1 & 1 & 0.76 & 1.58 & 0.08 & 30.98 \\
\hline & & $\%$ & $50 \%$ & $50 \%$ & & & & \\
\hline & Community hospital & Count & 69 & 56 & 0.45 & 1.36 & 0.61 & 3.03 \\
\hline & & $\%$ & $55 \%$ & $45 \%$ & & & & \\
\hline & Cancer Center & Count & 24 & 13 & 0.13 & 4.17 & 0.66 & 26.21 \\
\hline & & $\%$ & $65 \%$ & $35 \%$ & & & & \\
\hline & Total & Count & 333 & 265 & & & & \\
\hline & & $\%$ & $56 \%$ & $44 \%$ & & & & \\
\hline \multirow[t]{12}{*}{ Insurance } & Insurance through my job & Count & 215 & 183 & 0.96 & 0.98 & 0.53 & 1.81 \\
\hline & & $\%$ & $54 \%$ & $46 \%$ & & & & \\
\hline & Private insurance that I purchase on my own & Count & 20 & 18 & 0.88 & 1.07 & 0.44 & 2.58 \\
\hline & & $\%$ & $53 \%$ & $47 \%$ & & & & \\
\hline & Public insurance (Medicaid, Medicare) & Count & 57 & 32 & 0.70 & 1.16 & 0.54 & 2.51 \\
\hline & & $\%$ & $64 \%$ & $36 \%$ & & & & \\
\hline & None & Count & 4 & 3 & NA & NA & NA & NA \\
\hline & & $\%$ & $57 \%$ & $43 \%$ & & & & \\
\hline & Other & Count & 35 & 28 & 0.79 & 1.34 & 0.15 & 12.04 \\
\hline & & $\%$ & $56 \%$ & $44 \%$ & & & & \\
\hline & Total & Count & 331 & 264 & & & & \\
\hline & & $\%$ & $56 \%$ & $44 \%$ & & & & \\
\hline
\end{tabular}


Table 1 (continued)

\begin{tabular}{|c|c|c|c|c|c|c|c|c|}
\hline & & & \multicolumn{4}{|c|}{ Delay } & \multicolumn{2}{|c|}{$\begin{array}{l}95 \% \text { CI for } \\
\operatorname{Exp}(\beta)\end{array}$} \\
\hline & & & No & Yes & Sig & $\operatorname{Exp}(\beta)$ & Lower & Upper \\
\hline \multirow[t]{14}{*}{ Current cancer stage } & Stage 0 & Count & 23 & 18 & 0.36 & 1.80 & 0.51 & 6.36 \\
\hline & & $\%$ & $56 \%$ & $44 \%$ & & & & \\
\hline & Stage 1 & Count & 81 & 68 & 0.20 & 2.10 & 0.67 & 6.59 \\
\hline & & $\%$ & $54 \%$ & $46 \%$ & & & & \\
\hline & Stage 2 & Count & 95 & 82 & 0.20 & 2.12 & 0.68 & 6.61 \\
\hline & & $\%$ & $54 \%$ & $46 \%$ & & & & \\
\hline & Stage 3 & Count & 46 & 36 & 0.36 & 1.75 & 0.53 & 5.75 \\
\hline & & $\%$ & $56 \%$ & $44 \%$ & & & & \\
\hline & Stage 4 & Count & 75 & 54 & 0.29 & 1.87 & 0.59 & 5.96 \\
\hline & & $\%$ & $58 \%$ & $42 \%$ & & & & \\
\hline & Not sure/don't know & Count & 13 & 5 & NA & NA & NA & NA \\
\hline & & $\%$ & $72 \%$ & $28 \%$ & & & & \\
\hline & Total & Count & 333 & 263 & & & & \\
\hline & & $\%$ & $56 \%$ & $44 \%$ & & & & \\
\hline
\end{tabular}

Note: Total counts may differ slightly based on missing data

$46 \%, 44 \%$, and $42 \%$ for Stages $0,1,2,3,4$, respectively. The only variable which had a significant effect was age (97 $(95,99), p<0.001)$ with younger respondents $(M=45.94$, $S D=10.31)$ reporting a higher incidence of delays than older respondents $(M=48.98, S D=11.10)$. Characteristics of those who experienced delays compared with those who did not are presented in Table 1, along with odds ratios and confidence intervals.

Respondents reported the highest rate of delays in routine or follow-up clinic appointments (79\%), surgical breast reconstruction (66\%), diagnostic imaging (60\%), and lab testing (50\%). Respondents reported the lowest rate of delays in genetic counseling and testing (11\%) and oral therapies (13\%). Approximately $30 \%$ of respondents reported delays in hospital or clinic-based cancer therapies, including radiation $(30 \%)$, infusion therapies (32\%), and surgical tumor removal (26\%). Delay counts and proportions by type of care are presented in Table 2.

\section{Discussion}

The results of our study indicate that in the early weeks of the COVID-19 pandemic, nearly half of all breast cancer survivors experienced delays in cancer care. The data suggest that despite documented disparities in breast cancer outcomes and access to care based on factors such as race, insurance, site of care, and geography $[19,20]$, the pandemic response resulted in widespread barriers to breast cancer care regardless of other pre-existing healthcare system inequities. The consistency of delays across multiple factors also suggests that delays are not based on a strategic approach of risk-stratification in which we would expect to see higher rates of delays among patients with significant risk factors, for example, among patients who are immunocompromised or those with metastatic disease. The emerging literature points instead to ad hoc or reactive work-arounds that tried to balance shortages in medical resources and cancer survivors' risks of infection or disease progression [21, 22].

Significant disparities in treatment delays by age may be, in part, the result of delays in procedures or protocols specific to young women, particularly ovarian suppression. Some participants also reported delays in injections for ovarian suppression due to clinic closures or consolidation of clinical services in a different location, while others reported changes in their treatment protocol, including longer times between injections or an alternative therapy (e.g. tamoxifen) that did not require a clinic visit. These factors do not, however, explain all of the disparity in delays by age. The disparity is noteworthy for two reasons, and suggests an opportunity for further investigation. First, approximately $11 \%$ of individuals diagnosed with breast cancer in the US are under 45 years old [23, 24]. This population experiences more aggressive disease and 
Table 2 Delay counts and proportions by type of care $(n=263)$

\begin{tabular}{|c|c|c|c|}
\hline & & \multicolumn{2}{|l|}{ Delay } \\
\hline & & $\begin{array}{l}\text { No } \\
\text { Age }(M=48.98 \\
S D=11.10)\end{array}$ & $\begin{array}{l}\text { Yes } \\
\text { Age } \\
(M=45.94, \\
S D=10.31)\end{array}$ \\
\hline \multirow[t]{2}{*}{ Routine or follow-up clinic appointment } & Count & 51 & 191 \\
\hline & $\%$ & $21 \%$ & $79 \%$ \\
\hline \multirow[t]{2}{*}{ Lab testing } & Count & 89 & 89 \\
\hline & $\%$ & $50 \%$ & $50 \%$ \\
\hline \multirow[t]{2}{*}{ Diagnostic imaging or testing } & Count & 60 & 89 \\
\hline & $\%$ & $40 \%$ & $60 \%$ \\
\hline \multirow[t]{2}{*}{ Genetic testing or counseling } & Count & 49 & 6 \\
\hline & $\%$ & $89 \%$ & $11 \%$ \\
\hline \multirow[t]{2}{*}{ Surgery_mastectomy or lumpectomy } & Count & 42 & 15 \\
\hline & $\%$ & $74 \%$ & $26 \%$ \\
\hline \multirow[t]{2}{*}{ Surgery—breast reconstruction } & Count & 28 & 55 \\
\hline & $\%$ & $34 \%$ & $66 \%$ \\
\hline \multirow[t]{2}{*}{ Surgery—oophorectomy } & Count & 16 & 8 \\
\hline & $\%$ & $67 \%$ & $33 \%$ \\
\hline \multirow[t]{2}{*}{ Radiation } & Count & 35 & 15 \\
\hline & $\%$ & $70 \%$ & $30 \%$ \\
\hline \multirow[t]{2}{*}{ Infusion (e.g., chemo, immunotherapy) } & Count & 56 & 26 \\
\hline & $\%$ & $68 \%$ & $32 \%$ \\
\hline \multirow[t]{2}{*}{ Oral therapy } & Count & 116 & 18 \\
\hline & $\%$ & $87 \%$ & $13 \%$ \\
\hline \multirow[t]{2}{*}{ Other } & Count & 16 & 43 \\
\hline & $\%$ & $27 \%$ & $73 \%$ \\
\hline
\end{tabular}

Note: Respondents could choose as many categories as applicable higher rates of mortality. Second, data from the pandemic indicate that older people have a higher risk of mortality from COVID-19, with increased risk due to exposure during hospital or clinic-based treatment, as well as additional comorbidities. Despite these factors, our data suggest that older breast cancer survivors experienced significantly lower rates of delays in cancer treatment, with younger survivors bearing a disproportionate burden of treatment delays.

Our study results point to the pervasive impact of COVID-19 on cancer care, and a critical gap in disaster preparedness that leaves vulnerable cancer survivors at even greater risk for poor cancer outcomes, including not only clinical outcomes, but factors such as mental health, pain, and quality of life. As strategies emerge to address the backlog of patients whose care has been delayed, it is critical to recognize the differential impact of delays across the healthcare system, evidenced in part in the documented disparities in both breast cancer and COVID-19 mortality [25]. The factors underlying these disparities must be recognized and addressed in the development of systems and processes to deliver high quality cancer care to those whose care has been delayed to reduce the risk of exacerbating existing health inequities $[15,26]$ and ensure access to high quality cancer care for all breast cancer survivors.

Our study has several limitations. Due to the unprecedented circumstances of a global pandemic, no validated survey exists. Given the urgency of collecting real-time data, we were not able to validate this survey before distributing. Our national sample did not permit analysis of geographic differences based on rates of COVID-19 cases and the potential impact on cancer care in regions with higher rates of infection. Recruitment through social media may not be representative of all breast cancer survivors and may result in a selection bias with a higher response rate by younger survivors. This may also be a strength as it captured a segment of the cancer survivor population that is often underrepresented.

Acknowledgements We are grateful to numerous colleagues, patient advocates, community members, and friends who helped to distribute this survey (Dr. Deanna Attai, Mrs. Beulah Brent, Ms. Sophia Williams, Ms. Dikla Benzeevi, Ms. Ricki Fairley, Dr. Sasha Milicevic, Ms. Sarah Rittner, Ms. Christine Jonel, Ms. Jessica Roubitcheck, Ms. Tamika Felder, Ms. Kimberly Richardson, Sisters Working It Out, 
Metavivor Research and Support, Recovery on Water, Chicago Breasties, and the Breast Cancer Resource Center). Dr. Zhengjia Chen provided statistical support. The UIC Center for Clinical and Translational Science UL1TR002003 provided access to REDCap and associated technical support.

Funding This study was not funded.

\section{Compliance with ethical standards}

Conflict of interest The authors declare that they have no conflict of interest.

Ethical approval The study was reviewed by University of Illinois at Chicago's Institutional Review Board (Protocol \#2020-0371; Exemption Granted: 3/27/20). All procedures performed in this study involving human participants were in accordance with the ethical standards of the institutional and/or national research committee and with the 1964 Helsinki declaration and its later amendments or comparable ethical standards.

Informed consent This was an Internet-based study. Participants indicated consent by proceeding to and completing the survey.

\section{References}

1. Richards M, Anderson M, Carter P, Ebert BL, Mossialos E (2020) The impact of the COVID-19 pandemic on cancer care. Nat Cancer 1(6):565-567. https://doi.org/10.1038/s43018-020-0074-y

2. Kuderer NM, Choueiri TK, Shah DP et al (2020) Clinical impact of COVID-19 on patients with cancer (CCC19): a cohort study. Lancet. https://doi.org/10.1016/S0140-6736(20)31187-9

3. Printz C (2020) When a global pandemic complicates cancer care. Cancer 126(14):3171-3173. https://doi.org/10.1002/cncr.33043

4. Kutikov A, Weinberg DS, Edelman MJ, Horwitz EM, Uzzo RG, Fisher RI (2020) A war on two fronts: cancer care in the time of COVID-19. Ann Intern Med. https://doi.org/10.7326/M20-1133

5. Bartlett DL, Howe JR, Chang G et al (2020) Management of cancer surgery cases during the COVID-19 pandemic: considerations. Ann Surg Oncol. https://doi.org/10.1245/s10434-020-08461-2

6. Hooper MW, Nápoles AM, Pérez-Stable EJ (2020) COVID-19 and racial/ethnic disparities. JAMA 323(24):2466-2467. https:// doi.org/10.1001/jama.2020.8598

7. Gravlee C (2020) Racism not genetics, explains why Black Americans are dying of COVID-19. Scientific American Blog Network. https://blogs.scientificamerican.com/voices/racism-not-geneticsexplains-why-black-americans-are-dying-of-covid-19/. Accessed 17 July 2020

8. Mein SA (2020) COVID-19 and health disparities: the reality of "the Great Equalizer". J Gen Intern Med. https://doi.org/10.1007/ s11606-020-05880-5

9. Flume P, Gray S, Bowman CM, Kerrigan C, Lester M, VirellaLowell I (2005) Emergency preparedness for the chronically ill. Am J Nurs 105(3):68-72. https://doi.org/10.1097/00000446200503000-00028

10. Ford ES, Mokdad AH, Link MW et al (2020) Chronic disease in health emergencies: in the eye of the hurricane. Prev Chronic Dis. https://www.ncbi.nlm.nih.gov/pmc/articles/PMC1563956/. Accessed 20 March 2020

11. Ryan BJ, Franklin RC, Burkle FM Jr et al (2016) Reducing disaster exacerbated non-communicable diseases through public health infrastructure resilience: perspectives of Australian disaster service providers. PLoS Curr. https://doi.org/10.1371/currents.dis. d142f36b6f5eeca806d95266b20fed1f

12. Slama S, Kim H-J, Roglic G et al (2016) Care of non-communicable diseases in emergencies. Lancent. https://doi.org/10.1016/ S0140-6736(16)31404-0

13. Dhillon N, Castillo D'Andreis E, Boulmay BC (2015) Hepatocellular carcinoma (HCC) outcomes in a public hospital setting: characteristics and outcomes from the interim LSU public hospital (ILH) in New Orleans. Am Soc of Clin Oncol 33(15):e17533

14. McKinney N, Houser C, Meyer-Arendt K (2011) Direct and indirect mortality in Florida during the 2004 hurricane season. Int J Biometeorol 55(4):533-546

15. Man RX-G, Lack DA, Wyatt CE, Murray V (2018) The effect of natural disasters on cancer care: a systematic review. Lancet Oncol 19(9):e482-e499

16. Lai A G, Pasea L, Banerjee A et al (2020) Estimating excess mortality in people with cancer and multimorbidity in the COVID19 emergency. ResearchGate. https://www.researchgate.net/ publication/340984562_Estimating_excess_mortality_in_peopl e_with_cancer_and_multimorbidity_in_the_COVID-19_emerg ency. Accessed 27 May 2020

17. Harris PA, Taylor R, Minor BL et al (2019) The REDCap consortium: building an international community of software platform partners. J Biomed Inform 95:103208

18. Definition of survivor - NCI dictionary of cancer terms-National Cancer Institute. https://www.cancer.gov/publications/dictionari es/cancer-terms/def/survivor. Accessed 17 July 2020

19. Akinyemiju T, Moore JX, Ojesina AI, Waterbor JW, Altekruse SF (2016) Racial disparities in individual breast cancer outcomes by hormone-receptor subtype, area-level socio-economic status and healthcare resources. Breast Cancer Res Treat 157(3):575-586

20. Hoppe EJ, Hussain LR, Grannan KJ, Dunki-Jacobs EM, Lee DY, Wexelman BA (2019) Racial disparities in breast cancer persist despite early detection: analysis of treatment of stage 1 breast cancer and effect of insurance status on disparities. Breast Cancer Res Treat 173(3):597-602

21. Emanuel EJ, Persad G, Upshur R et al (2020) Fair allocation of scarce medical resources in the time of Covid-19. Mass Medical Soc. https://doi.org/10.1056/NEJMsb2005114

22. Rosenbaum L (2020) Harnessing our humanity-how Washington's health care workers have risen to the pandemic challenge. $\mathrm{N}$ Engl J Med. https://doi.org/10.1056/NEJMp2007466

23. Breast Cancer in Young Women. Centers for Disease Control and Prevention. https://www.cdc.gov/cancer/breast/young_women/ index.htm. Accessed 27 May 2020

24. Cancer Facts \& Figures 2020. American Cancer Society. https:// www.google.com/search?q=American+Cancer+Society.+Cance $\mathrm{r}+$ Facts $+\% 26+$ Figures $+2020 . \& \mathrm{rlz}=1 \mathrm{C} 1 \mathrm{GGRV}$ enUS756US7 $56 \&$ oq $=$ American + Cancer + Society + +Cancer + Facts $+\% 26+$ Figur es $+2020 . \&$ aqs $=$ chrome. $.69 \mathrm{i} 57 \mathrm{j} 013.1092 \mathrm{j} 0 \mathrm{j} 4 \&$ sourceid $=$ chrom e\&ie $=U T F-8$. Accessed 27 May 2020

25. van Dorn A, Cooney RE, Sabin ML (2020) COVID-19 exacerbating inequalities in the US. Lancet 395(10232):1243-1244

26. Schrag D, Hershman DL, Basch E (2020) Oncology practice during the COVID-19 pandemic. JAMA. https://doi.org/10.1001/ jama.2020.6236

Publisher's Note Springer Nature remains neutral with regard to jurisdictional claims in published maps and institutional affiliations. 